\title{
Análisis mediante Bio-Feedback, Adaptación Escolar y Intervención Neuroeducativa de un Caso de Autismo Grave
}

\author{
Bio-Feedback Analysis, School Adaptation and Neuroeducational Intervention in a \\ Case of Severe Autism
}

Agustín Ernesto Martínez-González ${ }^{1}$ y Juana López Gil ${ }^{2}$

\begin{abstract}
Resumen
El bio-feedback es una herramienta útil para evaluar la actividad del sistema nervioso y analizar el estado emocional de las personas con Trastorno del Espectro Autista (TEA). Existen pocos estudios que analicen las variables fisiológicas y la conducta adaptativa de las personas con TEA. El presente estudio tiene el objetivo de analizar las diferencias en la conducta agresiva, nivel de anticipación, gravedad de las conductas repetitivas, niveles de ansiedad y su relación con la adaptación escolar de un caso de autismo grave con discapacidad intelectual severa. El sujeto presenta niveles de cortisol altos y problemas en la microbiota intestinal. Los resultados indican un aumento de los niveles de ansiedad, gravedad de la conducta agresiva, estereotipias y autolesiones relacionadas con la inadaptación escolar. Se halla una disminución de respuesta fisiológica tras la intervención neuroeducativa. Futuros estudios deberán estudiar las relaciones entre variables fisiológicas, conductuales, gastro-intestinales y la eficacia de los tratamientos.
\end{abstract}

Palabras clave: autismo, biofeedback, adaptación escolar, ansiedad, intervención neuroeducativa

\begin{abstract}
Bio-feedback is a useful tool for assessing the activity of the nervous system and analyzing the emotional state of people with Autism Spectrum Disorder (ASD). There are few studies that analyze the physiological variables and the adaptive behavior of people with ASD. The present study aims to analyze the differences in aggressive behavior, anticipation level, severity of repetitive behaviors, anxiety level and its relation to the school adaptation of a case of severe autism with severe intellectual disability. The participant has high levels of cortisol and problems in the intestinal microbiota. The results indicate an increase in anxiety levels, aggressive behavior, stereotypies and self-injury related to school maladjustment. There is a decrease in physiological response after the neuroeducational intervention. Future studies should study the relationships between physiological, behavioral, gastro-intestinal variables and the efficacy of treatments.
\end{abstract}

Keywords: autism, biofeedback, school adaptation, anxiety, neuroeducational intervention

\footnotetext{
${ }^{1}$ Doctor en Psicología. Profesor Contratado Doctor. Departamento de Psicología Evolutiva y Didáctica. Facultad de Educación. Universidad de Alicante, España. Tel.: +34 965903400 x 2268. Correo: agustin.emartinez@ua.es. Orcid: 0000-0002-9576-7348

${ }^{2}$ Diplomada en magisterio d educación especial y grado en educación primaria. Tutora de centro de educación especial. Colegio Concertado de Educación Especial "Virgen de la Esperanza".
} 


\section{Introducción}

La literatura científica ha señalado la importancia de evaluar la agresividad (GonzálezPeña, Carrasco, del Barrio, \& Gordillo, 2013) y las funciones ejecutivas en el contexto escolar (Musso, 2009). Dentro del contexto de las necesidades educativas especiales encontramos al alumnado con trastorno del espectro autista (en adelante TEA). En el TEA hay una gran variedad de características que dependen tanto del nivel cognitivo como de la sintomatología que presenta la persona (Gabriela \& Buceta, 2012). Los criterios diagnósticos del TEA se centran en la severidad de los déficits en la comunicación social y los patrones repetitivos y restringidos de conductas. De tal modo que a mayor nivel de gravedad del TEA se requiere mayor supervisión y apoyo en la persona con TEA (Nivel 1: necesita ayuda; Nivel 2: necesita ayuda notable y Nivel 3: necesita ayuda muy notable). En este sentido, los niveles de gravedad en el TEA van a influir en la adaptación de la persona al contexto educativo (APA, 2013).

A lo largo de los últimos años investigaciones recientes han señalado la necesidad de analizar el origen de los estados emocionales negativos, como las conductas agresivas y las autolesiones en personas con TEA. Un estado emocional negativo en las personas con TEA puede ocasionar la aparición de problemas graves de conducta que interfieren en el funcionamiento escolar y relaciones sociales (Inada et al., 2015; Rojahn et., 2013). De hecho, existe una relación clara entre las conductas repetitivas y síntomas indicativos de mala adaptación al medio como la agresividad, la irritabilidad, y los problemas de conducta en las personas con TEA (Inada et al., 2015; Rojahn et., 2013; Scahill et al., 2014). Concretamente, los comportamientos estereotipados, los ritualísticos y de similitud son las conductas repetitivas más comunes en niños de preescolares con TEA (Fulceri et al., 2016) y que tienen un pronóstico negativo tanto en la adaptación como en la gravedad de los síntomas TEA (Martínez-González \& Piqueras, en prensa; Troyb et al., 2016). Un estudio reciente concluye que las conductas estereotipadas y las autolesiones son más graves en los colegios de educación especial (Martínez-González \& Piqueras, prensa).
Otros aspectos importantes a considerar son los antecedentes familiares y el tratamiento farmacológico. Los resultados de un estudio indica que el grupo de personas con TEA que tiene antecedentes psiquiátricos directos y el grupo de personas con TEA que reciben tratamiento farmacológico presentan puntuaciones significativamente más elevadas en comportamientos autolesivos (Martínez-González $\&$ Piqueras, 2017).

Así pues, hay ciertos factores que determinan la relación entre estados emocionales negativos y las dificultades en la adaptación escolar. El primer factor tiene relación con las dificultades de comunicación de las personas con TEA y su relación con la ansiedad. Autores sugiere que hay una disfunción en el sistema nervioso autónomo (SNA) de las personas con TEA y como consecuencia aparecen las conductas repetitivas (Condy, Scarpa, \& Friedman, 2017; Corbett, Muscatello, \& Baldinger, 2019). Además, en esta disfunción en el SNA interviene la rigidez cognitiva, que es la que determina la severidad de la conducta repetitiva, en concreto la conducta de similitud. La gravedad de la conducta de similitud esta positivamente correlacionada con la dificultad a la adaptación a los cambios y, por lo tanto, con la calidad de vida de la persona con TEA (Hollander, Taylor, Racine, Ferretti, \& Noone, 2016). Un segundo factor a considerar es la implicación de los problemas gastro-intestinales (estreñimiento, dolor abdominal, diarrea, etc.) y ciertas bacterias en los problemas emocionales y la conducta de las personas con TEA, sería el llamado eje intestino-cerebro (Andreo-Martínez, García-Martínez， \& Sánchez-Samper, 2017; Andreo-Martínez, García-Martínez, Quesada, Sánchez, \& Martínez-González, 2019; Ding, Taur, \& Walkup, 2017). En este sentido, las alteraciones cerebrales en el TEA (p.ej.: núcleo caudado, amígdala, hipotálamo y ganglios basales, entre otras estructuras cerebrales) son aspectos importantes a considerar en la etiología de las conductas compulsivas (Martínez-González, Piqueras, \& Pineda, 2016). El tercer factor es el hormonal, ya que se ha evidenciado una relación entre los intereses restringidos y los factores hormonales (Frazier \& Hardan, 2017). Así, parece ser que las conductas estereotipadas pueden ser un indicador de niveles de cortisol altos en personas 
con TEA (Lydon et al., 2015; Yang et al., 2015). Pero al mismo tiempo, estas conductas repetitivas tienen la función de mitigar los niveles de malestar o angustia de la persona con autismo, bajando los niveles de cortisol (Gabriels et al., 2013).

En el análisis del SNA de las personas con TEA, se han empleado herramientas como en biofeedback y el neurofeedback tanto como herramienta de evaluación como de intervención (Carrick et al., 2018; Goodman et al., 2018). El biofeedback es un instrumento preciso que permite medir la actividad fisiológica por medio de: ondas cerebrales, función cardiaca, la respiración, la actividad muscular, la temperatura y conductancia de la piel (Yucha \& Montgomery, 2008). Por otra parte, el neurofeedback, es un tipo de electroencefalografía o EEG, que entrena habilidades de autorregulación mediante tecnología computarizada (Hammond, 2011).

La literatura científica ha investigado los patrones respiratorios mediante el biofeedback para medir el funcionamiento del SNA y analizar su relación con las conductas repetitivas en personas con TEA. Así, las arritmias respiratorias son un predictor de la gravedad de las conductas repetitivas (Condy, Scarpa, \& Friedman, 2017). Por otra parte, es novedosa la utilización del biofeedback como herramienta de evaluación del SNA en personas con TEA (Sharma et al., 2017). De este modo, se obtiene información objetiva sobre el estado emocional de la persona con TEA que tiene problemas graves de comunicación (Betancourt, Dethorne, Karahalios, \& Kim, 2017). Además, otros estudios han empleado métodos específicos para la evaluación de la ansiedad en casos de autismo severo (Martínez-González \& López Gil, 2017).

Estudios recientes han señalado los beneficios del entrenamiento en autocontrol emocional con el biofeedbak y el neurofeedback. Los estudios señalan que estas herramientas pueden ayudar a generar cambios a nivel fisiológico y en la actividad cerebral (Yucha \& Montgomery, 2008). Estos cambios significativos en la actividad cerebral también se han evidenciado tanto a nivel fisiológico como conductual en niños con TEA (Carrick et al.,2018).

El presente estudio tiene el objetivo de analizar los estados emocionales negativos en un caso de TEA grave con discapacidad intelectual severa. En concreto se pretende analizar la frecuencia y tipología de la conducta, así como la respuesta fisiológica en dos situaciones relacionadas con la adaptación escolar (ir o no ir a casa el fin de semana) en un caso de autismo severo en régimen de residencia y colegio de educación especial. Así pues, los objetivos específicos de este estudio son analizar: 1) las diferencias en conducta agresiva y nivel de anticipación ante la situación de a ir o no a casa el fin de semana; 2) gravedad de las conductas repetitivas; 3) niveles de ansiedad y su relación con la adaptación escolar (ir o no ir a casa el fin de semana) tras la intervención neuroeducativa.

\section{Método}

\section{Descripción del caso}

Previa autorización de los padres describimos el contexto de este caso: J es un varón de 19 años de edad. $J$ es un chico que fue adoptado, procedente de Rumanía, a los 1 años de edad. Al poco tiempo de llegar a España se observaron los primeros síntomas de aislamiento social. Empezó a decir palabras sueltas a la edad de 3 años. Se evidenciaron problemas importantes en la psicomotricidad fina. Estuvo escolarizado en centro de educación especial. A los 10 años se realizaron varias pruebas neurofisiológicas una $\mathrm{R}$. Espectroscopia cerebral, una EEG de sueño y una ECG donde no se hallaron datos de alteraciones cerebrales. En la pubertad (11-12 años) $\mathrm{J}$ se encontraba más descontento e irritable y a los 14 años presentó por primera vez crisis de agitación psicomotriz con agresión física. J parecía ser más sensible a los sonidos ambientales muy fuertes y tiene molestias auditivas en la oreja derecha. Sin embargo, los episodios de agresividad eran difíciles de determinar; aunque según informes médicos estos episodios aparecían en situaciones de baja tolerancia a la frustración y otras veces eran debidos a molestias físicas relacionadas con el estómago. A los 14 años se le realizó una RMN cerebral hallándose focos de calcificación en ambos globos pálidos y quiste de retención en seno maxilar derecho. Igualmente, se le realizó en J una ecografía abdominal y los resultados estuvieron dentro de la normalidad. Sin embargo, se halló resultados positivos en sangre del 
antígeno de la bacteria helicobacter pylori. Desde entonces hasta la actualidad $\mathrm{J}$ ha presentado problemas gastro-intestinales (estreñimiento). A lo largo de estos años J había presentado muchos altibajos emocionales, sobre todo en cuanto a conducta, obsesiones y manías, que habían dificultado mucho la intervención educativa con él ya que las conductas disruptivas cada vez eran más frecuentes y de mayor intensidad, tanto en el colegio como en la casa y durante el transporte. J agredía a una persona del centro a la cual tenía condicionada. En el curso 2012-13 ingresó dos meses (octubre y noviembre) en el hospital y se le realizó un ajuste de la medicación (aripiprazol, ácido valproico, quetiapina y clorazepato). Informes de la Generalitat Valenciana (2013) califican a J con un $79 \%$ de discapacidad por Trastorno del desarrollo por Autismo etiología no filiada. Dada la gravedad de las conductas repetitivas de $\mathrm{J}$, los padres decidieron escolarizar a $\mathrm{J}$ en un colegio específico de educación especial que disponía de régimen de residencia (2013). Las evaluaciones realizadas tanto en el contexto del colegio como de la residencia (2013) con el Inventario de Espectro Autista (IDEA; Rivière, 2002), sugerían un nivel de autismo severo (colegio:51/ residencia:56). Los resultados de la Escala de conductas repetitivas - revisada (Repetitive Behavior Scale - Revised; RBS-R; Bodfish, Symons, Parker, \& Lewis, 2000; Martínez-González \& Piqueras, 2018) iban en la misma línea: Comportamiento estereotipado una puntuación 7 (Media española 3.7; Desviación típica 4.9); Comportamiento Autolesivo una puntuación de 4 ( $\mathrm{M}=1.6$; $\mathrm{DT}=3.5)$; Compulsivo una puntuación de $7 \quad(\mathrm{M}=1.4 / \quad \mathrm{DT}=1.6)$; Ritualístico una puntuación de $4 \quad(\mathrm{M}=3.2 /$ $\mathrm{DT}=3.7)$; Similitud una puntuación de $12(\mathrm{M}=3.5$; $\mathrm{DT}=4.4)$; Restringido una puntuación de 0 $(\mathrm{M}=1.6 ; \mathrm{DT}=1.9)$ y RBS-R Total una puntuación de $34(\mathrm{M}=15.3 ; \mathrm{DT}=15.2)$. Se concluye que la gravedad del comportamiento autolesivo, compulsivo y de similitud de $\mathrm{J}$ estaba por encima de la media española. Por otra parte, se hallaron puntuaciones por encima de la media, pero sin llegar a una desviación típica en comportamiento estereotipado. Los resultados del Inventory for Client and Agency Planning (ICAP; Bruininks, Hill, Weatherman, \& Woodcock, 1986; Montero, 1996) indicaban que $J$ presenta un nivel de conducta adaptativa de 5-6, lo que precisa una supervisión frecuente. En cuanto al nivel de apoyo, los resultados de la Escala de Intensidad de Apoyos (SIS; Verdugo, Ibáñez, \& Arias, 2007) mostraban que $\mathrm{J}$ tiene un índice de necesidad de apoyo muy severo (índice de necesidad de apoyo del 99\%). Por áreas se destaca que $\mathrm{J}$ requiere de un grado de supervisión constante en todas las actividades de la vida cotidiana (vida en el hogar; $99 \%$, aprendizaje a lo largo de la vida; $99 \%$, empleo; 99\%, salud y seguridad; 99\% y social; 99\%; vida en comunidad 99\%). Igualmente, necesitaba de una supervisión a nivel conductual esporádica (P.D:7). Respecto a la motricidad J presentaba buena motricidad gruesa, aunque las capacidades más específicas como la psicomotricidad, coordinación viso-espacial y motricidad fina estaban muy afectadas. En el año 2016 se realiza la primera valoración cognitiva hasta el momento, aplicando la Batería de Visualización y Razonamiento de la Escala Manipulativa Internacional de Leiter-Revisada (Roid \& Miller, 1996). Los resultados indicaban una puntuación compuesta de un CI no verbal breve de 36 y un CI Completo de 30. El informe técnico del Instituto Murciano de Acción Social (2016) califica a J con un $97 \%$ de minusvalía por Autismo y Retraso Mental Moderado. En el mismo año los resultados de laboratorio encontraron que $\mathrm{J}$ presentaba unos niveles de cortisol superiores a la media (Resultado: 20,4 $\mu \mathrm{g} / \mathrm{dL}$ siendo el intervalo de 6,2-19,4).

La medicación que tuvo prescrita durante la realización del presente estudio fue: ácido valproico $500 \mathrm{mg} \quad(1 / 0 / 0)$; palmitato de paliperidona $3 \mathrm{mg}$ (1/0/0); carbonato de litio (1/0,5/ 1); quetiapina 400mg (1/1/1); aripiprazol $15 \mathrm{mg}(1 / 0 / 0)$; aripiprazol $5 \mathrm{mg}(0 / 1 / 0)$; clonazepan $2 \mathrm{mg}(1,5 / 1 / 1)$; y si hay agresividad una dosis de 5 $\mathrm{mg}$ olanzapina y $2 \mathrm{mg}$ de clonazepan.

\section{Diseño}

Se siguió un diseño $\mathrm{AB}$ con mediciones previas, siendo la línea base de un mes y medio. El tratamiento comenzó pasado un mes y medio por haber un periodo de vacaciones, mientras que el postest se realizó tras cinco meses de intervención neuroeducativa. 


\section{Instrumentos}

Registro de conducta agresiva y anticipatoria: Instrumento elaborado ad-hoc para indicar la incidencia de conductas hetero-agresividad y anticipación a ir a casa durante la semana. Se evalúa en base a una escala tipo Thustone de 0 a 1 . Donde 0 significa no aparece la conducta y 1 significa que aparece la conducta.

Registro de conductas repetitivas: Instrumento elaborado ad-hoc basado algunas de las dimensiones del Repetitive Behavior Scale-Revised (RBS-R: Bodfish et al., 2000; Martínez-González \& Piqueras, 2018). El RBS-R es una escala que evalúa las conductas repetitivas mediante 43 ítems mediante seis dimensiones distintas de comportamiento repetitivo en los individuos con trastornos del espectro autista y discapacidad: a) Conductas Estereotipadas, b) Autolesivas, c) Compulsivas, d) Ritualísticas y e) Restricciones. El instrumento elaborado $a d-h o c$ evalúa la aparición de las conductas repetitivas basadas en las subescalas de Estereotipias y Autolesiones del RBS-R, siendo ambas las más implicadas en los problemas de conducta (Martínez-González \& Piqueras, prensa). En las conductas estereotipadas se observa la aparición de movimientos del cuerpo y estereotipias de los dedos. Por otra parte, se evalúa las conductas que indican búsqueda de estimulación sensorial. La evaluación de la aparición de las estereotipias, autolesiones y búsqueda sensorial se realizan en base a una escala tipo Thustone de 0 a 1 . Donde 0 significa no aparece la conducta y 1 significa que aparece la conducta.

Biofeedback: El biofeedback que se utilizó fue el eSense Skin Response de Mindfied (Mindfieldbiosystems, 2016-18). Este bio-feedback mide la conductancia de la piel a través de dos sensores situados en el índice y pulgar de la mano. Los sensores de conductancia de la piel van conectados a la toma de micrófono de una tablet (Android o Apple iOS). La actividad fisiológica se puede observar por medio de una aplicación específica llamada eSense App. El instrumento está diseñado para proporcionar medidas exactas y actuales de los niveles de ansiedad indicados en microsegundos ( $\mu \mathrm{s})$. Además, el eSense Skin Response puede ser utilizado para entrenar y controlar los niveles de ansiedad. La aplicación proporciona curvas de medición cuyos datos pueden ser exportados a una tabla Excel.

\section{Procedimiento}

Las sesiones de evaluación e intervención se realizan en el aula habitual de $\mathrm{J}$ y con la presencia de la tutora que era quien aplicaba todos los ítems del protocolo. Dada la gravedad de las conductas compulsivas $\mathbf{J}$ no estaba habituado a tener objetos que estuvieran adheridos a los dedos. Por lo que se requirió de un proceso de habituación de 6 semanas, dos sesiones a la semana de 6 minutos. En dicho proceso de habituación al bio-feedback se utilizan distractores musicales reforzantes.

Después de que $\mathrm{J}$ se halla adaptado al procedimiento en el aula, se realizó una línea base de un mes analizando la actividad fisiológica. Posteriormente, se inició la aplicación del protocolo de evaluación e intervención neuroeducativa. La aplicación del protocolo era de 10 minutos, siendo dos sesiones a la semana durante 5 meses. Las sesiones eran a primera hora de la mañana de 9:30 a 9:40 los viernes y lunes. Ello permitía tener una medida de los niveles de ansiedad antes de irse a la casa de sus padres, cada 15 días los viernes por la tarde, y cómo era el estado emocional a la llegada al colegio, ya que era el primer día de clase de la semana tras estar de fin de semana con los padres. En definitiva, estos datos proporcionan una visión global de la conducta adaptativa al centro educativo. El protocolo de intervención neuroeducativa contaba de dos partes diferenciadas: la primera parte constaba de varios ítems visuales y auditivos relacionados con emociones positivas y negativas, mientras que la segunda parte contaba con ítems de estimulación cognitiva (atencional y visoespacial mediante una tablet). El bio-feedback se aplicaba solamente a la primera parte del protocolo relacionada con el componente emocional. El objetivo de la primera parte del protocolo era estimular la empatía emocional de $\mathbf{J}$ y disminuir el número de agresiones en el colegio. Los ítems se elaboraron desde una perspectiva ecológica, es decir, las fotos y videos eran de los propios maestros y alumnos/as que expresaban emociones positivas o negativas. A continuación indicamos los ítems o estímulos visuales relacionados con el componente emocional: 1) Foto de un ojo herido por la agresión realizada a una alumna; 2) Hablar sobre esta alumna (se incita a identificar qué alumna es la que tiene la herida); 3) Foto de la tutora triste (se acompaña a la foto la 
expresión verbal de la emoción por parte de la tutora: "Si J pega yo estoy triste"); 4) Foto de la tutora contenta (se acompaña a la foto la expresión verbal de la emoción por parte de la tutora: "Si J no pega yo estoy contenta"); 5) Video de la alumna contenta y con volumen de voz alto (la tutora preguntará: “¿Cómo esta X? ¿Contenta o triste?"); 6) Video de la alumna contenta y con volumen de voz alto, segunda toma (la tutora dirá: "X está contenta, no se pega a X"); 7) Video de la alumna expresando deseo de amistad (la tutora dirá: "¿Qué quiere ser X? Quiere ser tu amiga"); 8) Video de la alumna contenta y con volumen de voz alto, tercera toma; y 9) Video de la alumna triste amistad (la tutora preguntará: “¿Cómo está X? Está triste"). Después de la aplicación del protocolo $\mathrm{J}$ tenía que realizar tareas de tipo atencional y visoespacial mediante una tablet con la supervisión de su maestra. En la última fase de la intervención neuroeducativa se realiza a $\mathrm{J}$ una exposición in vivo a una alumna que tiene condicionada y a la cual agrede con frecuencia, y se mide la actividad fisiológica con el biofeedback. En todas las fases de la intervención educativa se utilizaban indicadores visuales y un panel con las tareas a realizar para temporalizar las tareas.

\section{Análisis de datos}

Los análisis estadísticos se realizaron mediante el IBM SPSS Statistics v 24.0 for Windows (IBM SPSS Corp., Chicago, IL). Se realizaron el cálculo de los porcentajes en heteroagresividad y conducta repetitiva; y las medias de la respuesta fisiológica del eSense Skin Response de Mindfied (Mindfield-biosystems, 2016-18).

\section{Resultados}

\section{Conducta agresiva, anticipación y adaptación escolar}

La recopilación de los datos desde la línea base hasta la fase de intervención neuroeducativa (5 meses totales) indican un porcentaje de heteroagresividad mayor en $\mathrm{J}$ cuando éste ha estado en casa el fin de semana. Del mismo modo, $\mathrm{J}$ anticipa verbalmente irse a casa con más frecuencia (Véase la Figura 1).

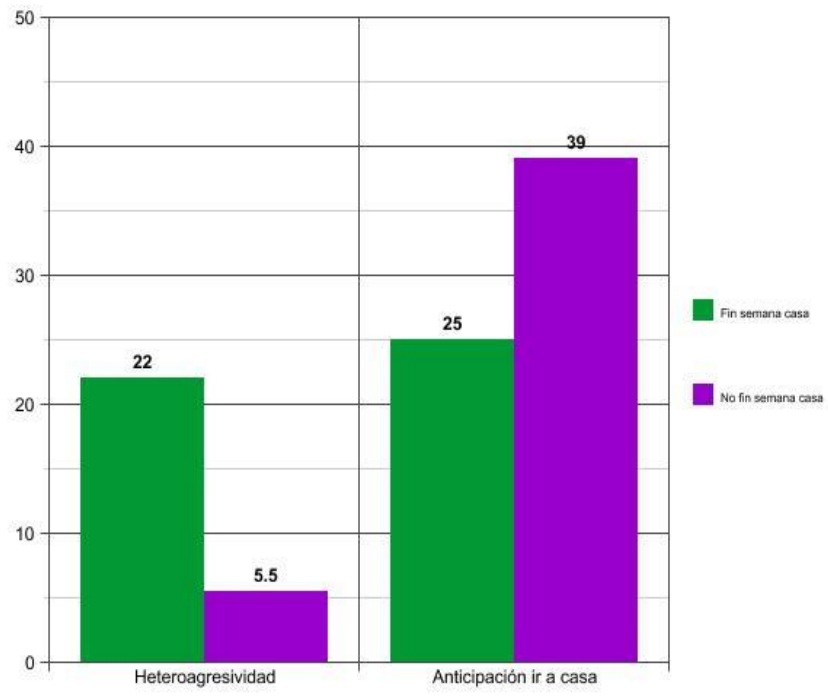

Figura 1. Porcentaje de aparición de heteroagresividad y anticipación a ir a casa según haber ido o no el fin de semana a casa

\section{Conducta repetitiva y adaptativa}

Los resultados sugieren diferencias clínicamente significativas en la frecuencia de las conductas repetitivas entre haber y no haber estado de fin de semana en casa. Concretamente, las diferencias más claras se observan en las conductas estereotipadas de movimientos del cuerpo (balanceo), conductas en busca de estimulación sensorial o en contacto corporal y autolesiones (Véase el Gráfico 2).

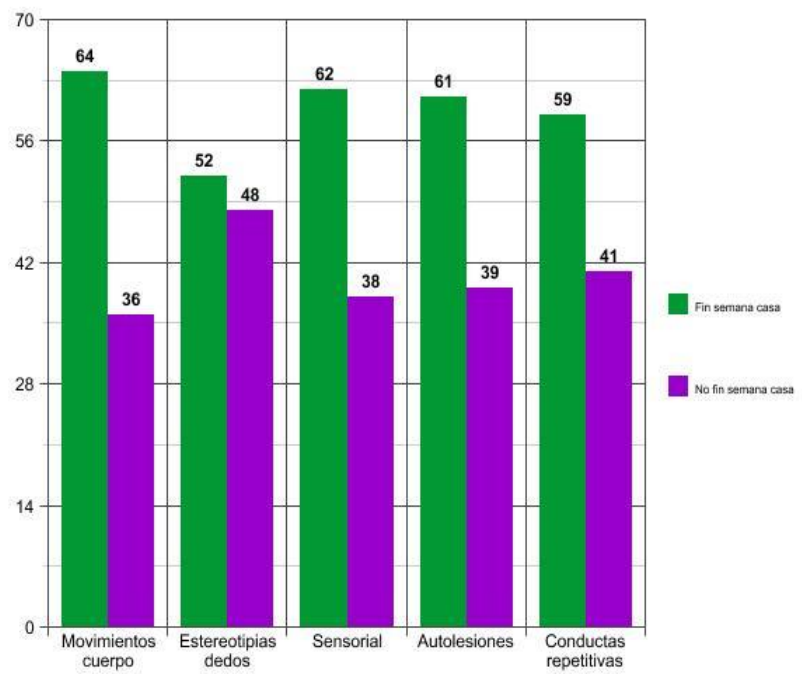

Figura 2. Porcentaje de aparición de conductas repetitivas según haber ido o no el fin de semana a casa

\section{Ansiedad, conducta adaptativa y efectos de la intervención neuroeducativa}

Los resultados de la evaluación fisiológica mediante biofeedback indican unos niveles más 
altos en la respuesta fisiológica o niveles de ansiedad indicados en microsegundos $(\mu s)$ en la semana que $\mathbf{J}$ se ha ido de fin de semana a casa respecto a la semana que no ha ido (Véase la Figura 3).

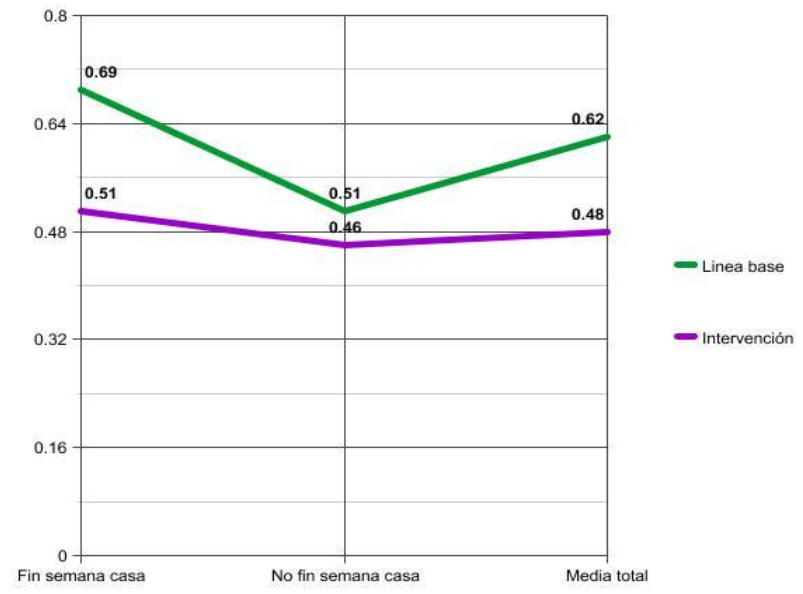

Figura 3. Diferencias en respuesta de ansiedad medida en microsegundos entre la linea base y la intervención neuroeducativa

Como puede observarse en la Figura 3 los resultados indican una disminución de la respuesta fisiológica tras la intervención neuroeducativa en los valores expresados en microsegundos tanto en la semana que $\mathrm{J}$ se fue de fin de semana a casa como la que no se fue a casa y la media total en microsegundos. Sin embargo, los resultados globales indican que los niveles de ansiedad son mayores cuando $\mathrm{J}$ vuelve de casa.

Concretamente, si se analizan los porcentajes de la tipología de curva fisiológica los resultados señalan un porcentaje mayor de curvas ascendentes de ansiedad cuando $\mathrm{J}$ vuelve de casa tanto en el primer mes como en los cuatro meses posteriores (Véase Figura 4).

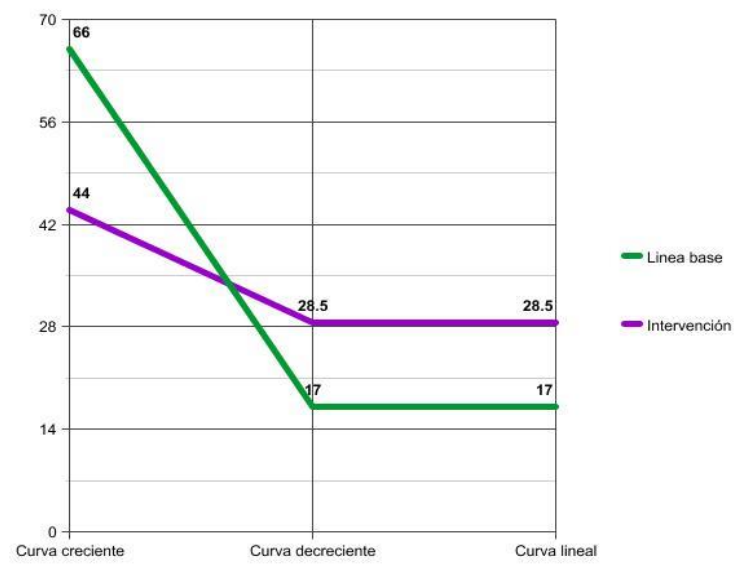

Figura 4. Diferencias en la tipología de curva fisiológica entre la línea base y la intervención neuroeducativa si $\mathrm{J}$ ha ido ese fin de semana a casa
Aunque el efecto de la intervención neuroeducativa tras cinco meses genera una disminución de la prevalencia en curvas ascendentes y un aumento de las curvas descendentes en la semana que el $\mathbf{J}$ ha estado el fin de semana en casa, las curvas ascendentes son las que tienen mayor prevalencia a nivel general durante todos estos meses de valoración en la situación de que el chivo vuelve de casa (véase Figura 4). Por lo tanto, este dato indica que sus niveles de ansiedad durante la semana de vuelta de casa son ascendentes.

Las semanas que $\mathrm{J}$ no ha ido de fin de semana a casa el primer mes o línea base indican que la mayoría de las curvas son de tipo ascendente, este resultado señala que la ansiedad de $\mathrm{J}$ va aumentando progresivamente durante la sesión de intervención en la clase (véase la Figura 5).

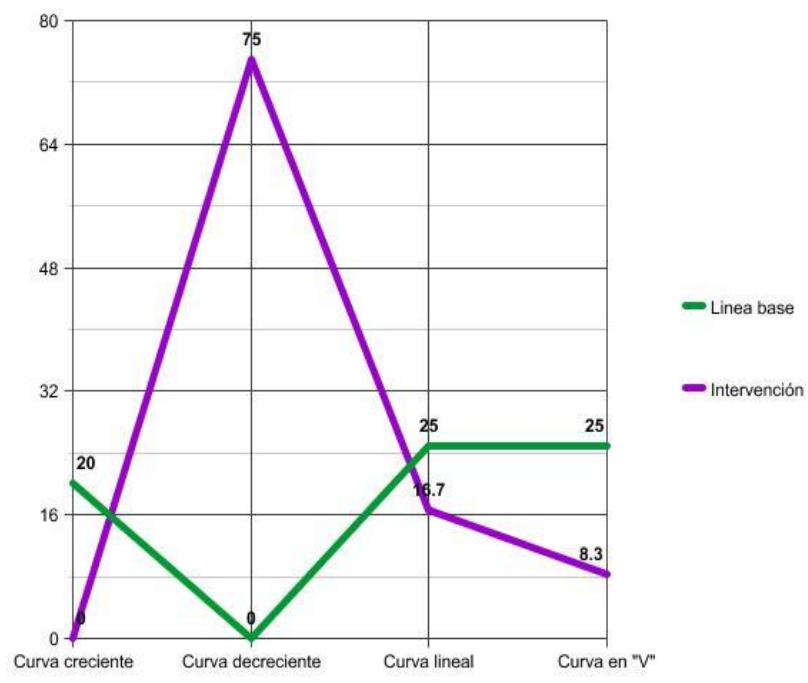

Figura 5. Diferencias en la tipología de curva fisiológica entre la línea base y la intervención neuroeducativa si $\mathrm{J}$ no ha ido ese fin de semana a casa

Tras cinco meses de intervención neuroeducativa se observa una disminución en las curvas de tipo ascendente de ansiedad y un aumento significativamente clínico en las curvas descendientes cuando $\mathbf{J}$ no se va el fin de semana a casa (Véase la Figura 5).

En la Figura 6 se muestra los datos de la última fase de la intervención. Se trata de las diferencias en la respuesta fisiológica entre la intervención neuroeducativa, basada en el desarrollo de la empatía emocional, y la exposición in vivo de $\mathrm{J}$ a una alumna que tiene condicionada y a la cual agrede con frecuencia. 
Del mismo modo, se analizan estos dos tipos de intervención con la variable haber ido o no el fin de semana a casa de los padres.

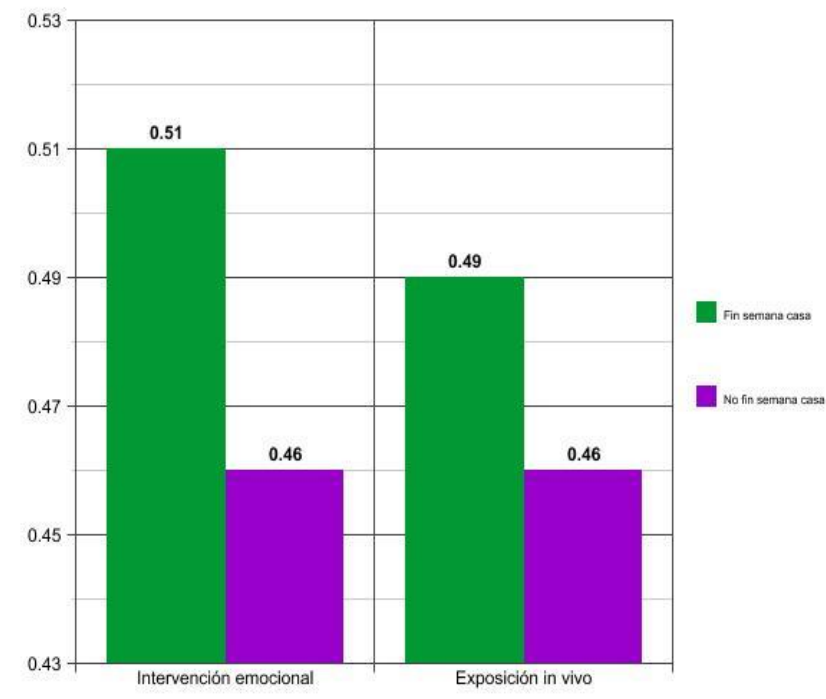

Figura 6. Diferencias en respuesta fisiológica entre intervención emocional y exposición in vivo según haber ido a casa el fin de semana

Los resultados indican que independientemente del tipo de intervención los niveles de ansiedad son más altos cuando $\mathrm{J}$ ha estado el fin de semana en casa. Aunque los niveles de ansiedad disminuyen entre la intervención emocional y la exposición in vivo, producto de la habituación.

\section{Discusión}

Habitualmente los educadores consideran que la anticipación de situaciones al alumno con TEA es fundamental para disminuir los niveles de estrés. Sin embargo, en el caso que se nos presenta la dimensión temporal de $\mathrm{J}$ esta preservada, sabe cuándo va a ir a casa ya que hay un aumento de la anticipación a ir a casa la semana que le toca ir. Por otra parte, a nivel de respuesta motora $\mathbf{J}$ presenta un aumento de las estereotipias, las autolesiones y busca con más frecuencia la estimulación sensorial o contacto corporal en su tutora y así como un aumento de la agresividad cuando viene de casa al colegio. Estos resultados son coincidentes con estudios anteriores que encuentran una mayor gravedad de las conductas repetitivas y en concreto de las autolesiones en personas que están en colegios de educación especial y residencias (MartínezGonzález \& Piqueras, prensa).
A nivel de respuesta fisiológica $J$ presenta unos mayores niveles de ansiedad cuando ha ido el fin de semana a casa. Parece ser que el biofeedack se muestra como una herramienta adecuada para detectar los niveles de ansiedad en personas con TEA que tienen dificultades de comunicación verbal (Betancourt et al., 2017). En el caso que nos ocupa esta herramienta es de gran utilidad dado que la persona presenta dificultades muy graves tanto en la comunicación como a nivel intelectual.

\section{Conclusiones}

Los resultados de nuestro estudio indican una disminución de la respuesta fisiológica y una mayor frecuencia de curvas descendentes de ansiedad tras la intervención neuroeducativa. Sin embargo, continúa habiendo un mayor porcentaje de curvas de ansiedad de tipo ascendente después de la intervención neuroeducativa cuando $\mathrm{J}$ ha ido a casa el fin de semana. Estos resultados son coherentes con los hallados en otros estudios que encuentran cambios a nivel fisiológico y de actividad cerebral en personas con TEA tras una intervención basada en el biofeedback y el neurofeedback (Carrick et al.,2018; Goodman et al., 2018). Sin embargo, la mayoría de los estudios encontrados son con población TEA y sin discapacidad intelectual.

Concluimos que los niveles de ansiedad, las conductas repetitivas y agresivas están asociados a una mayor frecuencia cuando $\mathrm{J}$ se ha ido de fin de semana a casa. El aumento de la conducta repetitiva, la anticipación verbal, sobre todo las autolesiones, y la búsqueda sensorial son signos de un aumento de excitación de activación del sistema nervioso simpático que conlleva la aparición de la conducta agresiva ulterior, como mecanismo de alivio que es difícil de controlar. Estos desajustes en los niveles de ansiedad dependiendo de la variable ir o no ir a casa sugieren dificultades graves de adaptación al centro. Además, J presenta diversos factores etiológicos además de los ambientales en la aparición de las emociones negativas, como son el desequilibrio hormonal, las alteraciones cerebrales y gastro-intestinales que coinciden con lo descrito en la literatura científica (Condy, Scarpa, \& 
Friedman, 2017; Corbett, Muscatello, \& Baldinger, 2019; Ding, Taur, \& Walkup, 2017; Hollander et al., 2016; Martínez-González, Piqueras, \& Pineda, 2016; Troyb et al., 2016).

Finalmente, a pesar que lo novedoso de este tipo de metodología de intervención en personas con TEA grave el presente estudio no está exento de limitaciones. En primer lugar, debido a que se trata de un caso $n=1$ no es posible realizar análisis estadísticos comparativos. Por lo tanto, futuros estudios deberán ampliar la muestra e incluir un grupo de control. En segundo lugar, aunque la comunidad científica reconoce que el método observacional mediante registro ad hoc para analizar las conductas es un método adecuado (Por ejemplo: Richardson et al., 2018). Sería deseable que en futuros estudios se pudieran incluir pruebas psicométricas para analizar los cambios emocionales. En tercer lugar, sería deseable aumentar el estudio a variables que relacionen el eje cerebro-microbiota y hormonas. En este sentido, futuros estudios tendrán que investigar la tipología de la ansiedad y el SNA de las personas con TEA y su relación con las repuestas repetitivas y las bacterias implicadas.

\section{Agradecimientos}

Quiéranos expresar nuestro sincero agradecimiento a la familia de este alumno con autismo por toda la confianza y colaboración que han prestado. Del mismo modo, quisiéramos dejar constancia de la gran profesionalidad de todos los trabajadores del CCEE "Virgen de la Esperanza". Sin ellos no hubiera sido posible la aplicación de estas nuevas herramientas de evaluación e intervención neuroeducativa.

\section{Referencias}

American Psychiatric Association (2013). Diagnostic and Statistical Manual of Mental Disorders (5a. ed.). Arlington, VA: American Psychiatric Publishing.

Andreo-Martínez, P., García-Martínez, N. G., \& Sánchez-Samper, E. P. (2017). El microbiota intestinal y su relación con las enfermedades mentales a través del eje microbiota-intestinocerebro. Revista de Discapacidad, Clínica y Neurociencias (RDCN), 4(2), 52-58. https://dialnet.unirioja.es/servlet/articulo?codi go $=6123886$

Andreo-Martínez, P., García-Martínez, N., Quesada, J., Sánchez, E., \& MartínezGonzález, A. E. (2019). Candida spp. en la microbiota intestinal de las personas con autismo: Revisión sistemática. Revista de neurología, 68(1), 1-6.

doi:10.33588/rn.6801.2018129

Betancourt, M. A., Dethorne, L. S., Karahalios, K., \& Kim, J. G. (2017). Skin conductance as an in situ marker for emotional arousal in children with neurodevelopmental communication impairments: Methodological considerations and clinical implications. $A C M$ Transactions on Accessible Computing (TACCESS), 9(3), 8. doi:10.1145/3035536

Bodfish, J. W., Symons, F. J., Parker, D. E., \& Lewis, M. H. (2000). Varieties of repetitive behavior in autism: Comparisons to mental retardation. Journal of Autism and Developmental Disorders, 30(3), 237-243. doi:10.1023/A:1005596502855

Carrick, F. R., Pagnacco, G., Hankir, A., Abdulrahman, M., Zaman, R., Kalambaheti, E. R., ... \& Oggero, E. (2018). The treatment of Autism Spectrum Disorder with auditory neurofeedback: A randomized placebo controlled trial using the mente autism device. Frontiers in neurology, 9, 537. doi:10.3389/fneur.2018.00537.

Condy, E. E., Scarpa, A., \& Friedman, B. H. (2017). Respiratory sinus arrhythmia predicts restricted repetitive behavior severity. Journal of Autism and Developmental Disorders, 47 (9), 2795-2804. doi:10.1007/s10803-017-3193-2

Corbett, B. A., Muscatello, R. A., \& Baldinger, C. (2019). Comparing stress and arousal systems in response to different social contexts in children with ASD. Biological psychology, 140 , 119-130. doi:10.1016/j.biopsycho.2018.12.010

Ding, H. T., Taur, Y., \& Walkup, J. T. (2017). Gut microbiota and autism: Key concepts and findings. Journal of Autism and Developmental Disorders, 47(2), 480-489. doi:0.1007/s10803-016-2960-9

Frazier, T. W., \& Hardan, A. Y. (20176). Equivalence of symptom dimensions in 
females and males with autism. Autism, 21 (6), 749-759. doi:10.1177/1362361316660066

Fulceri, F., Narzisi, A., Apicella, F., Balboni, G., Baldini, S., Brocchini, J., ... \& Tancredi, R. (2016). Application of the Repetitive Behavior Scale-Revised-Italian version-in preschoolers with autism spectrum disorder. Research in Developmental Disabilities, 48, 43-52. doi:10.1016/j.ridd.2015.10.015.

Gabriela-Nedelcu, D., \& Buceta, M. J (2012). El perfil cognitivo de los niños con trastorno de asperger y autismo de alto funcionamiento. Revista Iberoamericana de Diagnóstico y Evaluación - e Avaliação Psicológica 34(2), 103-116.

http://www.redalyc.org/html/4596/459645438 005/

Gabriels, R. L., Agnew, J. A., Pan, Z., Holt, K. D., Reynolds, A., \& Laudenslager, M. L. (2013). Elevated repetitive behaviors are associated with lower diurnal salivary cortisol levels in autism spectrum disorder. Biological Psychology, 93(2), 262-268. doi:10.1016/j.biopsycho.2013.02.017

González-Peña, P., Carrasco, M.A., del Barrio, V., \& Gordillo, R. (2013). Análisis de la agresividad reactiva y proactiva en niños de 2 a 6 años. Revista Iberoamericana de Diagnóstico y Evaluación e - Avaliação Psicológica, 35(1), 139-159. http://www.redalyc.org/html/4596/459645435 008/

Goodman, M. S., Castro, N., Sloan, M., Sharma, R., Widdowson, M., Herrera, E., \& Pineda, J. A. (2018). A neurovisceral approach to autism: Targeting self-regulation and core symptoms using neurofeedback and biofeedback. NeuroRegulation, 5(1), 9-29. doi:10.15540/nr.5.1.9.

Hammond, C. (2011). What is neurofeedback: An update. Journal of Neurotherapy, 15(4), 305336. http://dx.doi.org/10.1080/1087420 8.2011.623090.

Hollander, E., Taylor, B. P., Racine, E., Ferretti, C. J., \& Noone, R. (2016). Impulsivity and compulsivity: Translational approaches to compulsivity in autism spectrum disorders. European Neuropsychopharmacology, 26(5), 889-890. doi:10.1016/j.euroneuro.2015.06.021
IBM. (2013). SPSS Statistics for Windows (Version 22.0). [Computer software]. Armonk, NY: IBM Corp. Released

Inada, N., Ito, H., Yasunaga, K., Kuroda, M., Iwanaga, R., Hagiwara, T., ...\& Tsujii, M. (2015). Psychometric properties of the Repetitive Behavior Scale-Revised for individuals with autism spectrum disorder in Japan. Research in Autism Spectrum Disorders, $\quad 15, \quad 60-68$. doi:10.1016/j.rasd.2015.01.002

Lydon, S., Healy, O., Roche, M., Henry, R., Mulhern, T., \& Hughes, B. M. (2015). Salivary cortisol levels and challenging behavior in children with autism spectrum disorder. Research in Autism Spectrum Disorders, 10, 7892.doi:10.1016/j.rasd.2014.10.020

Martínez-González, A. E., \& López Gil, J. (2017). Un método para evaluar la ansiedad de un caso de autismo severo: Cambios tras la intervención. Revista de Discapacidad, Clínica y Neurociencias (RDCN), 4(2), 39-51. https://dialnet.unirioja.es/servlet/articulo?codi go $=6123885$

Martínez-González, A. E., \& Piqueras, J. A. (2017). Conductas repetitivas en personas con trastorno del espectro autista: relación con los antecedentes familiares y el tratamiento farmacológico. Revista de Educación Inclusiva, 10(1), 199-210. https://dialnet.unirioja.es/servlet/articulo?codi go=6049218

Martínez-González, A. E., \& Piqueras, J. A. (2018). Validation of the repetitive behaviour questionnaire in Spanish autism spectrum disorder. Journal of Autism and Developmental Disorders, 48(1),198-208. doi:10.1007/s10803-017-3276-0.

Martínez-González, A. E., \& Piqueras, J. A. (en prensa). Diferencias en la gravedad de los síntomas del Trastorno del Espectro Autista según el contexto educativo. European Journal of Education and Psychology.

Martínez-González, A. E., Piqueras, J. A., \& Pineda, D. (2016). Similitudes y diferencias en la sintomatología obsesivo-compulsiva y autista: Aportaciones desde la neurociencia. Revista Mexicana de Neurociencia, 17(5), 7082. 
http://www.medigraphic.com/pdfs/revmexneu /rmn-2016/rmn165g.pdf

Mindfield-biosystems (2016-18). eSense Skin Response. Germany.: Mindfield-biosystems. Recuperado de https://www.mindfield.de/en/

Musso, M. (2009). Evaluación de funciones ejecutivas en niños: Análisis y adaptación de pruebas en un contexto escolar. Revista Iberoamericana de Diagnóstico y Evaluación - e Avaliação Psicológica, 1(27), 157-178. http://www.redalyc.org/html/4596/459645443 009/

Richardson, K., Coeckelbergh, M., Wakunuma, K., Billing, E., Ziemke, T., Gomez, P., ... \& Belpaeme, T. (2018). Robot enhanced therapy for children with autism (DREAM): A social model of autism. IEEE Technology and Society Magazine, 37(1), 30-39. doi:10.1109/MTS.2018.2795096

Rivière, Á. (2002). IDEA: Inventario de espectro autista. Fundación para el desarrollo de los estudios cognitivos.

Roid, G. H., \& Miller, L. J. (1996). Escala manipulativa internacional de LeiterRevisada. Psymtec.

Rojahn, J., Schroeder, S. R., Mayo-Ortega, L., Oyama-Ganiko, R., LeBlanc, J., Marquis, J. \& Berke, E. (2013). Validity and reliability of the Behavior Problems Inventory, the Aberrant Behavior Checklist, and the Repetitive Behavior Scale-Revised among infants and toddlers at risk for intellectual or developmental disabilities: A multi-method assessment approach. Research in Developmental Disabilities, 34(5), 1804-1814. doi:10.1016/j.ridd.2013.02.024

Scahill, L., Dimitropoulos, A., McDougle, C. J., Aman, M. G., Feurer, I. D., McCracken, J. T., ...Vitiello, B. (2014). Children's Yale-Brown Obsessive Compulsive Scale in Autism Spectrum Disorder: Component structure and correlates of symptom checklist. Journal of the American Academy of Child and Adolescent Psychiatry, 53(1), 97-107. doi:10.1016/j.jaac.2013.09.018

Sharma, A., Sharma, A., Khosla, A., Khosla, A., Khosla, M., \& Khosla, M. (2017). Skin conductance response patterns of face processing in children with autism spectrum disorder. Advances in Autism, 3(2), 76-86. doi:10.1108/AIA-09-2016-0025

Troyb, E., Knoch, K., Herlihy, L., Stevens, M. C., Chen, C. M., Barton, M., ...\& Fein, D. (2016). Restricted and repetitive behaviors as predictors of outcome in autism spectrum disorders. Journal of Autism and Developmental Disorders, 46(4), 1282-1296. doi:10.1007/s10803-015-2668-2

Verdugo, M. A., Ibáñez, A., \& Arias, B. (2007). La Escala de Intensidad de Apoyos (SIS): Adaptación inicial al contexto español y análisis de sus propiedades psicométricas. Siglo Cero, 38(2), 5-16.

Yang, C. J., Tan, H. P., Yang, F. Y., Wang, H. P., Liu, C. L., He, H. Z., ... \& Du, Y. J. (2015). The cortisol, serotonin and oxytocin are associated with repetitive behavior in autism spectrum disorder. Research in Autism Spectrum Disorders, 18, 12-20. doi:10.1016/j.rasd.2015.07.002

Yucha, C., \& Montgomery, D. (2008). Evidencebased practice in biofeedback and neurofeedback. Wheat Ridge, CO: AAPB. 